\title{
VARIABLES ASOCIADAS A LOS MALOS TRATOS EN EL NOVIAZGO EN ADOLESCENTES Y ADULTOS JÓVENES
}

\author{
César Armando Rey-Anacona* \\ Grupo de Investigación en Psicología Clínica y de la Salud Universidad Pedagógica y Tecnológica de Colombia
}

Recibido, septiembre 1/2013

Concepto de evaluación, agosto 13/2014

Aceptado, noviembre 19/2014

\author{
Referencia: Rey-Anacona, C.A. (2015). Variables \\ asociadas a los malos tratos en el noviazgo en \\ adolescentes y adultos jóvenes. Acta Colombiana \\ de Psicología, 18(1), 159-171. DOI: 10.14718/ \\ ACP.2015.18.1.15
}

Resumen

\begin{abstract}
Se comparó un grupo de adolescentes y jóvenes adultos solteros y sin hijos que ejerció por lo menos una conducta de maltrato hacia su pareja, con un grupo similar que no realizó este tipo de maltrato. Las variables tenidas en cuenta fueron el número de actos de violencia observados entre los padres, el porcentaje de participantes de acuerdo con afirmaciones a favor de la violencia en el matrimonio y su puntuación en rasgos agresivos y comunicación de pareja. Asimismo, se realizó un análisis correlacional entre la frecuencia de malos tratos informados y las demás variables. Participaron 417 varones y 485 mujeres, entre 15 y 35 años. Los participantes del primer grupo reportaron haber presenciado más actos de violencia entre sus padres, estuvieron más de acuerdo con afirmaciones a favor de la violencia en el matrimonio y se adjudicaron más rasgos agresivos, variables que correlacionaron positivamente con la frecuencia de malos tratos. Estos resultados señalan que estas variables deberían contemplarse en la evaluación, el tratamiento y en los programas de prevención de la violencia en el noviazgo.

Palabras clave: violencia de pareja, adolescentes, adultos jóvenes, actitudes, personalidad
\end{abstract}

\section{VARIABLES ASSOCIATED WITH DATING VIOLENCE IN ADOLESCENTS AND YOUNG ADULTS}

\begin{abstract}
A group of teenagers and young single adults without children who had exercised at least one episode of abusive behavior towards their partner was compared with a similar group who had not carried out this type of abuse. The variables taken into account were the observed number of acts of violence between their parents, the percentage of participants who agreed with statements in favor of violence in marriage, and their score on aggressive traits and communication between members of the couple. Also, a correlation analysis between the frequency of reported abusive behavior and the other variables was performed. Participants were 417 men and 485 women between the ages of 15 and 35. Participants in the first group reported having witnessed more violence between their parents, agreed more with statements in favor of violence in marriage and acknowledged having more aggressive traits within themselves than participants in the second group. All of these variables showed positive correlations with the frequency of abusive behavior. These results indicate that these variables should be considered in assessment, treatment and prevention programs of dating violence.

Key words: Partner violence, adolescents, young adults, attitudes, personality
\end{abstract}

\footnotetext{
* Universidad Pedagógica y Tecnológica de Colombia, Facultad de Ciencias de la Salud, Escuela de Psicología, Calle 24 No. 5-63, Tel. (57 8) 7448585. Antiguo Hospital San Rafael, Tunja, Colombia. cesar.rey@uptc.edu.co

Este proyecto fue aprobado y recibió apoyo financiero de la Dirección de Investigaciones de la Universidad Pedagógica y Tecnológica de Colombia. Código: SGI-458. Las siguientes personas aplicaron instrumentos en esta investigación: Fredy Acero, Yesica Áriza, Mónica Bayona, Paola Bayona, Tatiana Castellanos, Ibeth Guerrero, Jenny López, Rosalba Mariño, Arturo Martínez, Sara Martínez, Maritza Mateus, Pedro Mojica, Lorena Monguí, Nidia Otálora, Lizzet Paitán, Jenniffer Pineda, Michael Pita, María Paula Pulido, Jennifer Ramírez, Víctor Rodríguez, Yeffer Roncancio y Alejandra Sosa.
} 


\title{
VARIÁVEIS ASSOCIADAS AOS MAUS TRATOS NO NAMORO EM ADOLESCENTES E ADULTOS JOVENS
}

\begin{abstract}
Resumo
Comparou-se um grupo de adolescentes e jovens adultos solteiros e sem filhos que exerceu pelo menos uma conduta de maltrato ao seu casal, com um grupo similar que não realizou este tipo de maltrato. As variáveis levadas em consideração foram o número de atos de violência observados entre os pais, a porcentagem de participantes de acordo com afirmações a favor da violência no casamento e sua pontuação em rasgos agressivos e comunicação de casal. Também, foi feita uma análise correlacional entre a frequência de maus tratos informados e as outras variáveis. Participaram 417 homens e 485 mulheres, entre 15 e 35 anos. Os participantes do primeiro grupo informaram haver presenciado mais atos de violência entre seus pais, estiveram mais de acordo com afirmações a favor da violência no casamento e apresentaram mais rasgos agressivos, variáveis que correlacionaram positivamente com a frequência de maus tratos. Estes resultados assinalam que estas variáveis deveriam ser contempladas na avaliação, no tratamento e nos programas de prevenção da violência no namoro.

Palavras chave: violência de casal, adolescentes, adultos jovens, atitudes, personalidade.
\end{abstract}

\section{INTRODUCCIÓN}

En los últimos años se ha incrementado el interés por los factores de riesgo de los actos de maltrato y violencia que ocurren en las relaciones de pareja de adolescentes y jóvenes que no se encuentran casados ni en convivencia (Centers for Disease Control and Prevention, 2006; Corral, 2009; Lewis \& Fremouw, 2001; Matud, 2007). En general, los resultados señalan que aquellos que fueron maltratados en su familia de origen, presenciaron violencia entre sus padres y presentan actitudes a favor de dicha violencia, están en mayor riesgo de ser victimarios o víctimas de malos tratos por parte de su pareja (e. g., Carlson, 1990; Foshee, Bauman \& Linder, 1999; Fredland et al., 2005; Kinsfogel \& Grych, 2004; Sears, Byers \& Price, 2007; Smith, Winokur \& Palenski, 2005). Sears et al. (2007), por ejemplo, encontraron en 633 adolescentes varones y mujeres de 12 a 18 años de edad, que las actitudes a favor de la violencia, la relación con pares que habían ejercido malos tratos hacia su pareja, el haber sido objeto de malos tratos por parte de la pareja y el temor a la violencia en la familia de origen, se relacionan significativamente con la ejecución de malos tratos físicos, psicológicos y sexuales. También hallaron que el número de adolescentes que había ejercido malos tratos físicos, psicológicos y sexuales era mayor conforme se incrementaba el grado de escolaridad.

En Iberoamérica se observa un interés similar por este tema con la publicación de algunos trabajos sobre las posibles consecuencias y los factores de riesgo de la violencia en el noviazgo. Rivera, Allen, Rodríguez, Chávez \&Lazcano (2006) examinaron las conductas de riesgo asociadas a la victimización entre 4.587 estudiantes femeninas de 12 a 24 años de edad seleccionadas aleatoriamente de las escuelas públicas de la Provincia de Morelos (México). Encontraron que el maltrato se relacionaba significativamente con depresión, consumo de tabaco, abuso de alcohol, bajo rendimiento escolar y antecedentes de relaciones sexuales. Saldivia \&Vizcarra (2012), similarmente, en un estudio entre 205 estudiantes universitarios chilenos de ambos géneros, concluyeron que el consumo de alcohol correlacionaba significativamente con el hecho de ser víctima de violencia física y psicológica. Por su parte, Muñoz-Rivas, GámezGuadix, Graña \& Fernández (2010) informaron que en un estudio entre 1.282 varones y mujeres de 15 a 20 años, el consumo elevado de alcohol y drogas ilegales incrementaba significativamente la probabilidad auto-informada de agredir física y sexualmente a la pareja, y que los participantes con un elevado consumo de estas sustancias reportaban iniciar más frecuentemente las agresiones hacia su pareja.

Vizcarra \&Póo (2011), por su parte, encontraron entre 427 estudiantes universitarios chilenos seleccionados aleatoriamente, que el recibir violencia física se relacionaba con la violencia psicológica recibida, actitudes favorables hacia la violencia y baja participación religiosa, mientras que el hecho de haber recibido violencia psicológica se asociaba con actitudes favorables hacia la violencia y un mayor tiempo de relación de pareja. Este último resultado es similar al reportado por Rey-Anacona (2009a), quien encontró una correlación positiva estadísticamente significativa entre el tiempo de relación y la frecuencia de malos tratos de tipo físico y psicológico, entre 403 adolescentes y adultos jóvenes.

En general, estos resultados son coherentes con los hallazgos de otras investigaciones que tienden a revelar factores de riesgo distales como la observación de violencia, los malos tratos en la familia de origen y la justificación de la violencia, al igual que otros proximales como el uso de bebidas alcohólicas y drogas ilegales, así como posibles consecuencias de los malos tratos como depresión, ingesta de bebidas alcohólicas y otras sustancias, y bajo rendimiento 
académico (Ackard \& Neumark-Sztainer, 2002; Centers for Disease Control and Prevention, 2006; Howard \& Wang, 2003; Kreiter et al., 1999; Roberts, Auinger \& Klein, 2005; Silverman, Raj, Mucci \& Hathaway, 2001).

Basado en un enfoque de aprendizaje social, en esta investigación se examinó la relación entre un conjunto de posibles factores de riesgo de la ejecución de malos tratos en el noviazgo, conforme a la revisión de la literatura y el esquema de factores de riesgo presentado en Rey-Anacona (2008a). Según éste, las experiencias de violencia en la familia de origen, el conocimiento de la realización de actos de maltrato por parte de pares conocidos y las experiencias precedentes con otras parejas, legitimarían el uso de la violencia hacia la pareja. Esto explicaría la presentación conjunta de la observación de violencia y la justificación de la violencia informada en algunos estudios sobre malos tratos en el noviazgo (e. g., Sears et al., 2007; Vizcarra \& Póo, 2011), así como los hallazgos de varias investigaciones que han señalado una relación entre haber presenciado violencia entre los padres y los malos tratos en el noviazgo (e. g., Ackard \& Neumark-Sztainer, 2002; Carr \& Vandeusen, 2002; Foshee et al., 1999; Gagné, Lavoie \& Hébert, 2005; Kinsfogel \& Grych 2004; Rich, Gidycz, Warkentin, Lohc \& Weiland, 2005; Rivera, Allen, Rodríguez, Chávez \& Lazcano, 2007; Sears et al., 2007; Wolfe, Scott, Wekerle $\&$ Pittman, 2001). En un estudio longitudinal realizado con adolescentes, Lichter \& McCloskey (2004), encontraron que aquellos que presenciaron violencia conyugal en su infancia justificaban más el uso de violencia en el noviazgo, lo que refuerza la idea de que dicha observación legitimaría el uso de la violencia con las propias parejas desde la adolescencia.

A su vez, la exposición a este tipo de violencia constituiría un modelo de resolución de conflictos que los individuos tenderían a repetir en sus relaciones de pareja, tal como se ha señalado en cuanto a la violencia marital (Browne \& Herbert, 1997; Mihalic \& Elliot, 1997). Por consiguiente, es de esperar menores habilidades de comunicación de pareja entre los individuos que tienden a ejercer malos tratos con su pareja, y un estilo agresivo de resolución de conflictos desde la adolescencia. Foshee et al. (1999), en ese sentido, encontraron una relación entre la ejecución de actos de violencia de pareja entre 1.965 estudiantes varones y mujeres de octavo y noveno grado, y haber presenciado o haber sido objeto de violencia en su familia, la cual estaba mediada por la aceptación del uso de la violencia y por un estilo agresivo de resolución de conflictos. Igualmente, Kinsfogel \& Grych (2004) encontraron entre 391 adolescentes de 14 a 18 años, que aquellos que habían presenciado mayor conflicto interparental, veían con mayor naturalidad la agresión en las relaciones románticas, tenían mayores dificultades para manejar la ira y creían que la violencia era común en las relaciones de pareja de sus pares.

Con el fin de obtener mayor evidencia empírica referente a si estos aspectos podrían ser factores de riesgo de los malos tratos en el noviazgo, dentro del esquema teórico señalado, esta investigación se planteó los siguientes objetivos:

- Comparar a un grupo de adolescentes y adultos jóvenes que ejercieron algún tipo de maltrato hacia su pareja en el noviazgo con un grupo similar que no ejerció este tipo de violencia, en relación con el número de actos de violencia observados entre los padres, el porcentaje de participantes de acuerdo con afirmaciones a favor de la violencia en el matrimonio, y su puntuación en rasgos agresivos y comunicación de pareja.

- Examinar la correlación entre la frecuencia de malos tratos ejercida hacia la pareja y estas variables.

\section{MÉTODO}

\section{Participantes}

Participaron 902 estudiantes vinculados a una universidad pública colombiana, 417 varones (46.2\%) y 485 mujeres (53.8\%), entre 15 y 35 años de edad $(X=19.32$ años; DT $=2.33$ años). Estos estudiantes vivían en barrios de estrato socioeconómico bajo bajo $(\mathrm{n}=43,4.8 \%)$, bajo $(\mathrm{n}=312$, $34.6 \%)$, medio bajo $(\mathrm{n}=384,42.6 \%)$, medio $(\mathrm{n}=120$, $13.3 \%)$, medio alto $(n=20,2.2 \%)$ y alto $(n=2,0.2 \%)$ y se encontraban cursando entre el primer y el noveno semestres de 22 carreras profesionales y de licenciatura que se ofrecen en la universidad mencionada, con una media de 2.37 semestres cursados.

\section{Instrumentos}

Lista de Chequeo de Experiencias de Maltrato en la Pareja (Rey-Anacona, 2009b). Es un cuestionario que permite informar la frecuencia de realización de 95 conductas de maltrato hacia la pareja, de tipo físico, verbal, emocional, psicológico, sexual, económico y negligente, por medio de una escala tipo Likert (opciones: "Nunca", "Una vez", "Algunas veces" o "Muchas veces"). Los últimos 13 ítems deben ser respondidos por personas que han conformado un hogar o han tenido hijos con su pareja, por lo que no fueron considerados en esta investigación. Este instrumento fue revisado a nivel metodológico y de contenido por jueces, obteniendo un alfa de Cronbach de .96. El análisis factorial con rotación varimax y el método de análisis de 
componentes principales mostró que el $50.54 \%$ de la varianza podría explicarse por seis factores (Rey-Anacona, Mateus-Cubides \& Bayona-Arévalo, 2010).

Cuestionario de Auto informe sobre Incidentes de Violencia Conyugal (Rey-Anacona, 2008b). Permite reportar 14 formas de violencia realizadas por parte del padre hacia la madre y las mismas 14 de la madre hacia el padre, para un total de 28 ítems que se contestan con un formato "Sí-No". Los tipos de violencia contemplados en este cuestionario son la violencia física, la violencia verbal, las amenazas verbales, las amenazas con un objeto contundente, encerrar a la persona en la casa o en un cuarto, las prohibiciones, echar de la casa a la otra persona, la violencia sexual, la violencia económica y las amenazas a personas cercanas. El cuestionario también permite informar con una pregunta abierta, otras formas de violencia ejercidas por parte del padre hacia la madre y viceversa, lo que da lugar a un total de 30 preguntas. Este instrumento fue revisado a nivel metodológico y de contenido por jueces expertos, presentando un alfa de Cronbach de .86 (Rey-Anacona, 2008b).

Inventario de Masculinidad y Feminidad ([IMAFE]; Lara, 1993). Es un cuestionario auto-descriptivo de la personalidad con base en 60 adjetivos que se responden a través de una escala Likert de siete opciones: "Nunca o casi nunca soy así" a "Siempre o casi siempre soy así". Permite evaluar cuatro patrones de personalidad de género: Masculinidad, Feminidad, Machismo y Sumisión, los cuales conforman cuatro escalas compuestas por 15 reactivos cada una. Los ítems de este instrumento fueron obtenidos a partir de los reactivos del Inventario de Papeles Sexuales de Bem (1974).

Los adjetivos de la escala de Machismo, la única que se utilizó en esta investigación, hacen referencia a rasgos de agresividad, dominación e intransigencia: Agresivo(a), Dominante, Autoritario(a), Rudo(a), Egoísta, Enérgico(a), De voz fuerte, Incomprensivo(a), Uso malas palabras, Individualista, Materialista, Arrogante, Frío(a), Ambicioso(a) y Malo(a). La escala de Masculinidad, por su parte, comprende adjetivos referentes a rasgos considerados masculinos y positivos, asociados a lo práctico y a la orientación hacia la acción, mientras que la escala de Feminidad incluye rasgos considerados femeninos positivos, asociados con la relación, las habilidades interpersonales, la preocupación por los demás y la expresividad, mientras que la escala de Sumisión abarca rasgos considerados femeninos y negativos, relacionados con la abnegación, la dependencia, la subordinación y la debilidad (Lara, 1993).

La validación original de este instrumento se basó en los datos obtenidos con 1.301 mexicanos varones y mujeres, quienes mostraron tres factores que explicaban el $33.3 \%$ de la varianza (Lara, 1993). Los índices de confiabilidad obtenidos oscilaban entre .74 y .92 y las escalas correlacionaron significativamente con sus similares del Inventario de Roles Sexuales de Bem (1974). En Colombia, Martínez-Gómez, Guerrero-Rodríguez y Rey-Anacona (2012) evaluaron su confiabilidad y validez de constructo, por medio de los datos obtenidos con 1.527 varones y mujeres entre 15 y 42 años, informando valores alfa que oscilaban entre .76 y .88 . El análisis factorial mostró que los ítems se agrupaban alrededor de tres factores que explicaban el $30.8 \%$ de la varianza, congruentes con los encontrados en su validación original.

Cuestionario sobre Aceptación del Uso de la Violencia en el Matrimonio (Rey-Anacona, 2009b). El instrumento original permite manifestar el acuerdo o desacuerdo con 14 enunciados referentes al uso de la violencia por parte del esposo hacia la esposa, siendo validado a nivel metodológico por dos jueces y a nivel de contenido por tres jueces expertos en violencia en la familia (Rey-Anacona, 2008b). En esta investigación se incluyeron otros 14 ítems correspondientes a los 14 originales, pero en relación con la conducta de la esposa hacia su esposo. El alfa de Cronbach obtenido fue .89 (Rey-Anacona, 2009b).

Cuestionario de Comunicación Afectiva (Arroyo \& Vergara, 2000, adaptado por Rey-Anacona, 2008b). Este instrumento fue creado originalmente para evaluar la comunicación de pareja de los y las adolescentes entre 14 y 18 años de edad que han tenido o tienen una relación de noviazgo igual o superior a los dos meses, comprendiendo 30 ítems que se contestan a través de una escala numérica de cinco opciones. La puntuación arrojada por el instrumento refleja la presencia de dos habilidades de comunicación de pareja: la habilidad para manifestar afecto positivo y la habilidad para manifestar sentimientos negativos. ReyAnacona (2008b) lo sometió a un proceso de revalidación metodológica y de contenido, en un estudio en el que participaron 211 adolescentes bogotanos de 14 a 18 años de edad (101 varones y 110 mujeres), en el que se le realizaron varias mejoras a nivel de su escala de calificación, la redacción y calificación de algunos ítems y el tiempo de duración de la relación de pareja, obteniendo un alfa de .79 y un índice en la prueba de dos mitades estándar de .73. Además, el instrumento correlacionó con varias medidas teóricamente relacionadas (Vg., de habilidades sociales y de empatía), lo cual indica que es confiable y válido a nivel de constructo. En esta investigación mostró un alfa de .87.

\section{Procedimiento}

Debido al objetivo de la investigación, los participantes fueron seleccionados incidentalmente, solicitando en los salones de clase la colaboración de los estudiantes de 
Tabla 1.

Definición de variables

\begin{tabular}{|c|c|c|c|}
\hline Variable & Tipo & $\begin{array}{l}\text { Categorías/Unidad } \\
\text { de medida }\end{array}$ & Instrumento \\
\hline $\begin{array}{l}\text { Frecuencia de conductas de mal- } \\
\text { trato hacia la pareja }\end{array}$ & De razón & $\begin{array}{l}\text { Frecuencia promedio de } \\
\text { conductas reportadas }\end{array}$ & $\begin{array}{l}\text { Lista de Chequeo de Experiencias } \\
\text { de Maltrato en la Pareja (Rey-Ana- } \\
\text { cona, 2009) }\end{array}$ \\
\hline $\begin{array}{l}\text { Número de conductas de violencia } \\
\text { presenciadas entre los padres }\end{array}$ & De razón & $\begin{array}{l}\text { Número total de conductas } \\
\text { reportadas }\end{array}$ & $\begin{array}{l}\text { Cuestionario de Auto informe sobre } \\
\text { Incidentes de Violencia Conyugal } \\
\text { (Rey-Anacona, 2008b) }\end{array}$ \\
\hline $\begin{array}{l}\text { Características de personalidad } \\
\text { relacionadas con la agresividad, la } \\
\text { dominación y la intransigencia }\end{array}$ & De intervalo & $\begin{array}{l}\text { Puntuación en la Escala } \\
\text { de Machismo }\end{array}$ & $\begin{array}{l}\text { Inventario de Masculinidad } \\
\text { y Feminidad (Lara, 1993) }\end{array}$ \\
\hline \multirow{2}{*}{$\begin{array}{l}\text { Aceptación del uso de la violencia } \\
\text { en el matrimonio }\end{array}$} & \multirow{2}{*}{$\begin{array}{l}\text { Nominal } \\
(28 \text { ítems })\end{array}$} & 1 = Sí (Acuerdo) & \multirow{2}{*}{$\begin{array}{l}\text { Cuestionario sobre Aceptación del } \\
\text { Uso de la Violencia en el Matrimo- } \\
\text { nio (Rey-Anacona, 2008b) }\end{array}$} \\
\hline & & $2=$ No $($ Desacuerdo $)$ & \\
\hline $\begin{array}{l}\text { Número de acuerdos con enuncia- } \\
\text { dos a favor de la violencia en el } \\
\text { matrimonio }\end{array}$ & De razón & $\begin{array}{l}\text { Número de enunciados a favor } \\
\text { de la violencia en el matrimonio } \\
\text { respondidos con "Sí" }\end{array}$ & $\begin{array}{l}\text { Cuestionario sobre Aceptación del } \\
\text { Uso de la Violencia en el Matrimo- } \\
\text { nio (Rey-Anacona, 2008b) }\end{array}$ \\
\hline Comunicación de pareja & De intervalo & Puntuación directa & $\begin{array}{l}\text { Cuestionario de Comunicación } \\
\text { Afectiva (Arroyo \& Vergara, 2000, } \\
\text { adaptado por Rey-Anacona, 2008b). }\end{array}$ \\
\hline
\end{tabular}

los cursos programados para los primeros cinco semestres de las carreras mencionadas previamente, escogiéndose aquellos que hubieran tenido al menos una pareja sentimental ("novio" o "novia") y que fueran solteros y $\sin$ hijos. Estos participantes se dividieron entre aquellos(as) que reportaron haber ejercido al menos una conducta de maltrato a su pareja y los que no, en la Lista de Chequeo de Experiencias de Maltrato en la Pareja (Rey-Anacona, 2009b),y se compararon en las variables objeto de estudio.

Las baterías de instrumentos contestadas por los 902 participantes se incorporaron en una base de datos de SPSS versión 20.0. Debido a que las pruebas de normalidad Kolmogorov-Smirnov y Shapiro-Wilk evidenciaron que la muestra no se distribuía normalmente en las variables bajo análisis, con significancias superiores a $p=.001$, se efectuaron análisis estadísticos no paramétricos a nivel general y por sexo, aceptándose un nivel de significancia mínimo de .05 (bilateral). Las variables bajo estudio se definieron operacionalmente conforme a la información de la tabla 1.

\section{RESULTADOS}

En primera instancia se presentan los análisis estadísticos implementados y a continuación los resultados de dichos análisis. Los análisis estadísticos fueron los siguientes:
1. Se comparó el grupo de participantes que ejerció al menos una conducta de maltrato sobre su pareja, con el grupo de participantes que no informó este tipo de conductas, con respecto a las siguientes variables: a) El número de conductas violentas que presenció entre sus padres, b) sus puntuaciones en Machismo y c) el número de enunciados del Cuestionario sobre Aceptación del uso de la violencia en el matrimonio (Rey-Anacona, 2009b) con los que estaban de acuerdo. Para esto se utilizó la prueba U de Mann Whitney, y se calculó el tamaño del efecto con la prueba no parámetrica Cliff's Delta Calculator (Macbeth, Razumiejczyk \& Ledesma, 2001).

2. Se analizó la correlación entre la frecuencia de malos tratos ejercidos sobre la pareja con las demás variables mencionadas, a través de la prueba de Spearman, para lo cual se codificó dicha frecuencia de la siguiente manera: "Nunca"=0, "Una vez"=1, "Algunas veces"= 2 y "Muchas veces" $=3$.

3. Se comparó con el Chi Cuadrado, el porcentaje de participantes que ejerció al menos una conducta de maltrato sobre su pareja con el porcentaje de participantes que no ejerció este tipo de conductas, en relación con el número de integrantes que estaban de acuerdo con cada uno de los enunciados del Cuestionario sobre Aceptación del uso de la violencia en el matrimonio (Rey-Anacona, 2009b). 
4. Se comparó con la misma prueba estadística, el porcentaje de participantes que ejerció al menos una conducta de maltrato sobre su pareja con el porcentaje de participantes que no ejerció este tipo de conductas, en relación con el número de integrantes que presenciaron cada una de las 30 conductas de violencia que se presentan en el Cuestionario de Auto informe sobre incidentes de violencia conyugal (Rey-Anacona, 2008b).

A continuación se presentan los resultados obtenidos siguiendo el orden de los análisis estadísticos anteriormente enunciados.

\section{Diferencias entre los dos grupos de participantes}

En la tabla 2 se presentan los resultados de las comparaciones efectuadas entre el grupo que ejerció al menos una conducta de maltrato a su pareja (Grupo VN), con el grupo que no informó esta circunstancia (Grupo NVN), las cuales mostraron que los primeros presenciaron un número significativamente mayor de conductas de violencia entre sus padres comparados con los participantes del Grupo NVN, tendencia que se observó entre los varones aunque con un tamaño del efecto entre bajo y moderado $(d=0.37)$, así como entre las mujeres con un tamaño del efecto bajo $(d=0.26)$.

Tabla 2.

Diferencias entre el grupo que ejerció al menos una conducta de maltrato a su pareja (Grupo VN), con el grupo que no informó esta circunstancia (Grupo NVN)

\begin{tabular}{|c|c|c|c|c|c|c|}
\hline \multirow{3}{*}{ Variable } & \multicolumn{4}{|c|}{ General } & \multirow{3}{*}{$\mathrm{U}$} & \multirow{3}{*}{$\mathrm{P}$} \\
\hline & \multicolumn{2}{|c|}{$\mathrm{VN}$} & \multicolumn{2}{|c|}{ NVN } & & \\
\hline & $\mathrm{X}$ & DX & $\mathrm{X}$ & DX & & \\
\hline No. conductas de violencia presenciadas entre los padres & 2.50 & 3.42 & 1.19 & 2.76 & $\begin{array}{l}34748.5 \\
(\mathrm{n}=902)\end{array}$ & .000 \\
\hline Rasgos de agresividad/dominación/ intransigencia & 3.24 & 0.89 & 2.94 & 0.91 & $\begin{array}{l}40202.5 \\
(\mathrm{n}=901)\end{array}$ & .000 \\
\hline $\begin{array}{l}\text { No. acuerdos con enunciados a favor de la violencia } \\
\text { en el matrimonio }\end{array}$ & 5.56 & 4.92 & 4.40 & 4.69 & $\begin{array}{l}39512.0 \\
(\mathrm{n}=902)\end{array}$ & .000 \\
\hline Comunicación afectiva de pareja & 114.17 & 15.95 & 115.34 & 18.48 & $\begin{array}{l}47765.5 \\
(\mathrm{n}=902)\end{array}$ & .380 \\
\hline
\end{tabular}

\begin{tabular}{|c|c|c|c|c|c|c|}
\hline \multirow{3}{*}{ Variable } & \multicolumn{4}{|c|}{ Varones } & \multirow{3}{*}{$\mathrm{U}$} & \multirow{3}{*}{$\mathrm{P}$} \\
\hline & \multicolumn{2}{|c|}{$\mathrm{VN}$} & \multicolumn{2}{|c|}{ NVN } & & \\
\hline & $\mathrm{X}$ & DX & $\mathrm{X}$ & $\mathrm{DX}$ & & \\
\hline No. conductas de violencia presenciadas entre los padres & 2.52 & 3.68 & 0.75 & 1.82 & $\begin{array}{l}6245.0 \\
(\mathrm{n}=417)\end{array}$ & .000 \\
\hline Rasgos de agresividad/dominación/ intransigencia & 3.43 & 0.87 & 3.23 & 0.82 & $\begin{array}{l}8752.0 \\
(\mathrm{n}=417)\end{array}$ & .157 \\
\hline $\begin{array}{l}\text { No. acuerdos con enunciados a favor de la violencia en el } \\
\text { matrimonio }\end{array}$ & 6.28 & 5.63 & 5.42 & 5.66 & $\begin{array}{l}8321.0 \\
(\mathrm{n}=417)\end{array}$ & .048 \\
\hline Comunicación afectiva de pareja & 113.13 & 16.43 & 111.33 & 18.3 & $\begin{array}{c}9349.0 \\
(\mathrm{n}=417)\end{array}$ & .467 \\
\hline
\end{tabular}

\begin{tabular}{|c|c|c|c|c|c|c|}
\hline \multirow{3}{*}{ Variable } & \multicolumn{4}{|c|}{ Mujeres } & \multirow{3}{*}{$\mathrm{U}$} & \multirow{3}{*}{$\mathrm{P}$} \\
\hline & \multicolumn{2}{|c|}{$\mathrm{VN}$} & \multicolumn{2}{|c|}{ NVN } & & \\
\hline & $\mathrm{X}$ & DX & $\mathrm{X}$ & DX & & \\
\hline No. conductas de violencia presenciadas entre los padres & 2.48 & 3.17 & 1.52 & 3.25 & $\begin{array}{l}11349.5 \\
(\mathrm{n}=485)\end{array}$ & .017 \\
\hline Rasgos de agresividad/ dominación/ intransigencia & 3.07 & 0.86 & 2.74 & 0.92 & $\begin{array}{l}11569.5 \\
(\mathrm{n}=484)\end{array}$ & .001 \\
\hline $\begin{array}{l}\text { No. acuerdos con enunciados a favor de la violencia en el } \\
\text { matrimonio }\end{array}$ & 4.92 & 4.10 & 3.65 & 3.69 & $\begin{array}{l}11705.5 \\
(\mathrm{n}=485)\end{array}$ & .001 \\
\hline Comunicación afectiva de pareja & 115.08 & 15.47 & 118.28 & 18.17 & $\begin{array}{l}13406.0 \\
(\mathrm{n}=485)\end{array}$ & .078 \\
\hline
\end{tabular}

Nota: X: Media; DX: Desviación típica; U: Valor U de Mann Whitney; p: Probabilidad; n: Tamaño de la sub-muestra. 
El grupo VN también presentó puntuaciones significativamente mayores en rasgos de agresividad, dominación e intransigencia, tendencia que también se observó entre los dos grupos de mujeres con un tamaño del efecto bajo $(d=0.24)$, aunque no entre los varones $(d=0.11)$. Asimismo, el grupo $\mathrm{VN}$ evidenció un número significativamente mayor de acuerdos con enunciados referentes al uso de violencia en el matrimonio, comparado con el grupo NVN, tendencia que también se encontró entre los varones aunque con un tamaño del efecto bajo $(d=0.16)$ y entre las mujeres $(d=0.23)$. No se encontraron diferencias estadísticamente significativas en las puntuaciones en comunicación de pareja obtenido por ambos grupos, ni tampoco entre los dos grupos de varones $(d=0.06)$, ni los dos grupos de mujeres $(d=-0.12)$.

Correlaciones entre la frecuencia de malos tratos hacia la pareja y las demás variables

El análisis correlacional mostró que la frecuencia de malos tratos hacia la pareja correlacionaba positivamente con el número de conductas violentas presenciadas entre los padres $\left(r_{S}=.251, \mathrm{p}=.000\right)$, la puntuación en rasgos de personalidad de Agresividad/Dominación/Intransigencia $\left(r_{S}=.261, \mathrm{p}=.000\right)$ y el número de enunciados a favor del uso de la violencia en el matrimonio $\left(r_{S}=.260, \mathrm{p}=\right.$ $.000)$, y negativamente por la puntuación en comunicación de pareja $\left(r_{S}=-.173, \mathrm{p}=.000\right)$. Lo mismo ocurrió cuando se evaluaron estas correlaciones solamente con los participantes varones, entre quienes el número de conductas violentas entre los padres $\left(r_{S}=.286, \mathrm{p}=.000\right)$, la puntuación en los rasgos mencionados $\left(r_{S}=.232, \mathrm{p}=.000\right)$ y el número de enunciados a favor del uso de la violencia en el matrimonio $\left(r_{S}=.277, \mathrm{p}=.000\right)$, correlacionaron positivamente la frecuencia de malos tratos ejercidos a la pareja, mientras que con la comunicación afectiva de pareja correlacionó negativamente $\left(r_{S}=-.180, \mathrm{p}=.000\right)$. Entre las mujeres se obtuvieron resultados similares ya que el número de conductas violentas entre los padres $\left(r_{S}=.220, \mathrm{p}\right.$ $=.000)$, la puntuación en los rasgos mencionados $\left(r_{S}=.274\right.$, $\mathrm{p}=.000)$ y el número de enunciados a favor del uso de la violencia en el matrimonio $\left(r_{S}=.236, \mathrm{p}=.000\right)$ correlacionaron positivamente con la frecuencia de los malos tratos ejercidos hacia la pareja, mientras que la comunicación afectiva de pareja correlacionó negativamente con dicha frecuencia $\left(r_{S}=-.162, \mathrm{p}=.000\right)$. Sin embargo, aunque estas correlaciones fueron estadísticamente significativas, la fuerza de asociación evidenciada en éstas fue baja, ya que no superaron el valor de .3.
Malos tratos hacia la pareja y aceptación del uso de la violencia en el matrimonio

En cuanto a la aceptación del uso de la violencia en el matrimonio, las comparaciones realizadas con respecto al porcentaje de participantes del grupo VN y el Grupo NVN, que estuvo de acuerdo con cada una de las 28 afirmaciones que se presentan en el Cuestionario sobre Aceptación del Uso de la Violencia en el Matrimonio, mostró que los participantes que ejercieron alguna conducta de violencia a su pareja estuvieron más frecuentemente de acuerdo con ocho de estas 28 afirmaciones (véase Tabla 3).

Estas mismas comparaciones realizadas solamente entre los participantes varones no revelaron diferencias estadísticamente significativas entre los dos grupos. Entre las mujeres, por su parte, se encontró que las pertenecientes al grupo VN estuvieron más frecuentemente de acuerdo con las siguientes afirmaciones: (a) "En ciertas situaciones se justifica que el esposo grite a su esposa" $\left(X^{2}[1,481]\right.$ $=5.688, \mathrm{p}=.018)$; (b) "En ciertas situaciones se justifica que la esposa grite a su esposo" $\left(X^{2}[1,480]=8.083, \mathrm{p}=\right.$ .004); (c) "Es comprensible que una esposa insulte a su esposo si éste no ha cumplido con sus deberes como padre" $\left(X^{2}[1,481]=6.686, p=.01\right)$ y (d) "En ciertas situaciones se justifica que la esposa le prohíba a su esposo el trato con alguna persona, como un amigo o una amiga" $\left(X^{2}[1,482]\right.$ $=5.548, \mathrm{p}=.018$ ).

Malos tratos hacia la pareja y violencia entre los padres

En la tabla 4 se compara el porcentaje de participantes del grupo VN y del grupo NVN, que reportaron haber presenciado cada una de las 30 conductas de violencia entre los padres que se presentan en el Cuestionario de Auto informe sobre Incidentes de Violencia Conyugal. Los datos que aparecen en dicha tabla muestran que los participantes que ejercieron alguna conducta de maltrato hacia su pareja informaron con mayor frecuencia haber presenciado once de estas 30 conductas de violencia entre los padres.

Entre los varones se encontró que los del grupo VN reportaron con mayor frecuencia la observación de las siguientes conductas: a) Actos de violencia física (del padre a la madre $)\left(\mathrm{X}^{2}[1,417]=5.22, \mathrm{p}=.023\right)$; b) Groserías, insultos o humillaciones (por lo menos una vez por semana) (del padre a la madre) $\left(\mathrm{X}^{2}[1,417]=7.966, \mathrm{p}=.005\right)$; $\mathrm{c}$ Groserías, insultos o humillaciones (por lo menos una vez por semana) (de la madre al padre) $\left(\mathrm{X}^{2}[1,417]=9.737\right.$, $\mathrm{p}=.001)$; d) Prohibirle que trabaje o que estudie (de la madre al padre $)\left(\mathrm{X}^{2}[1,416]=4.399, \mathrm{p}=.036\right)$; e) Echarla de la casa o cerrarle la puerta para que no pudiese entrar (del padre a la madre) $\left(\mathrm{X}^{2}[1,417]=5.467, \mathrm{p}=.018\right)$; (f) Obligarlo a tener relaciones sexuales (de la madre al padre) $\left(\mathrm{X}^{2}[1,417]=4.386, \mathrm{p}=.035\right)$ y $(\mathrm{g})$ Negarse a darle plata 
Tabla 3.

Diferencias en el porcentaje de participantes que estaban de acuerdo con cada una de las afirmaciones a favor del uso de la violencia en el matrimonio

\begin{tabular}{|c|c|c|c|c|}
\hline Afirmación & $\begin{array}{l}\text { Ejerció } \\
\text { maltrato }\end{array}$ & No ejerció & $\mathrm{X}^{2}$ & $\mathrm{p}$ \\
\hline 1. En ciertas situaciones se justifica que el esposo grite a su esposa & $178(23.2 \%)$ & $16(12.4 \%)$ & 7.564 & $.005 * *$ \\
\hline 2. En ciertas situaciones se justifica que el esposo le pegue a su esposa & $40(5.2 \%)$ & $6(4.6 \%)$ & .082 & 1.000 \\
\hline 3. La infidelidad de la esposa es un motivo justificable para que su esposo le pegue & $105(13.7 \%)$ & $11(8.5 \%)$ & 2.714 & .119 \\
\hline 4. Es un delito que el esposo golpee a su esposa & $652(85.1 \%)$ & $110(84.6 \%)$ & .022 & .894 \\
\hline $\begin{array}{l}\text { 5. Es comprensible que un esposo insulte a su esposa si ésta no ha cumplido con sus deberes } \\
\text { como madre }\end{array}$ & $191(24.9 \%)$ & $20(15.4 \%)$ & 5.630 & $.019^{*}$ \\
\hline $\begin{array}{l}\text { 6. Es comprensible que un esposo insulte a su esposa si ésta no ha cumplido con sus deberes } \\
\text { como cónyuge }\end{array}$ & $98(12.8 \%)$ & $13(10 \%)$ & .791 & .471 \\
\hline $\begin{array}{l}\text { 7. Es comprensible que un esposo le pegue a su esposa si ésta no ha cumplido con sus deberes } \\
\text { como madre }\end{array}$ & $56(7.3 \%)$ & $8(6.2 \%)$ & .221 & .853 \\
\hline $\begin{array}{l}\text { 8. Es comprensible que un esposo le pegue a su esposa si ésta no ha cumplido con sus deberes } \\
\text { como cónyuge }\end{array}$ & $46(6 \%)$ & $8(6.2 \%)$ & .005 & 1.000 \\
\hline 9. La esposa debe obedecer las órdenes de su esposo, ya que éste es el jefe de familia & $71(9.3 \%)$ & $9(6.9 \%)$ & .759 & .505 \\
\hline $\begin{array}{l}\text { 10. El esposo está en el derecho de prohibirle a su esposa que trabaje o que estudie, si considera } \\
\text { que así ella puede dedicarse mejor al hogar y/o a los hijos }\end{array}$ & $49(6.4 \%)$ & $7(5.4 \%)$ & .191 & .845 \\
\hline $\begin{array}{l}\text { 11. En ciertas situaciones se justifica que el esposo le prohíba a su esposa el trato con alguna } \\
\text { persona, como un amigo o una amiga }\end{array}$ & $214(28 \%)$ & $21(16.2 \%)$ & 8.060 & $.005 * *$ \\
\hline $\begin{array}{l}\text { 12. Es comprensible que un esposo eche de la casa a su esposa si ésta no ha cumplido con sus } \\
\text { deberes como madre }\end{array}$ & $107(14 \%)$ & $16(12.4 \%)$ & .223 & .782 \\
\hline $\begin{array}{l}\text { 13. Es comprensible que un esposo eche de la casa a su esposa si ésta no ha cumplido con sus } \\
\text { deberes como cónyuge }\end{array}$ & $66(8.6 \%)$ & $10(7.7 \%)$ & .122 & .865 \\
\hline 14. Es una obligación de la esposa satisfacer sexualmente a su esposo & $18(20.7 \%)$ & $20(15.4 \%)$ & 1.936 & .191 \\
\hline 15. En ciertas situaciones se justifica que la esposa grite a su esposo & $257(33.6 \%)$ & $25(19.2 \%)$ & 10.567 & $.001 * * *$ \\
\hline 16. En ciertas situaciones se justifica que la esposa le pegue a su esposo & $50(6.5 \%)$ & $9(6.9 \%)$ & .031 & .848 \\
\hline 17. La infidelidad del esposo es un motivo justificable para que su esposa le pegue & $125(16.3 \%)$ & $12(9.2 \%)$ & 4.268 & $.047^{*}$ \\
\hline 18. Es un delito que la esposa golpee a su esposo & $559(73.1 \%)$ & $93(72.1 \%)$ & .054 & .831 \\
\hline $\begin{array}{l}\text { 19. Es comprensible que una esposa insulte a su esposo si éste no ha cumplido con sus deberes } \\
\text { como padre }\end{array}$ & $216(28.2 \%)$ & $25(19.2 \%)$ & 4.512 & $.033^{*}$ \\
\hline $\begin{array}{l}\text { 20. Es comprensible que una esposa insulte a su esposo si éste no ha cumplido con sus deberes } \\
\text { como cónyuge }\end{array}$ & $113(14.8 \%)$ & $14(10.8 \%)$ & 1.449 & .277 \\
\hline $\begin{array}{l}\text { 21. Es comprensible que una esposa le pegue a su esposo si éste no ha cumplido con sus debe- } \\
\text { res como padre }\end{array}$ & $72(9.4 \%)$ & $10(7.8 \%)$ & .360 & .624 \\
\hline $\begin{array}{l}\text { 22. Es comprensible que una esposa le pegue a su esposo si éste no ha cumplido con sus debe- } \\
\text { res como cónyuge }\end{array}$ & $40(5.2 \%)$ & $8(6.2 \%)$ & .184 & .673 \\
\hline 23. El esposo debe obedecer las órdenes de su esposa, ya que ésta es la jefa de familia & $60(7.8 \%)$ & $7(5.4 \%)$ & .970 & .373 \\
\hline $\begin{array}{l}\text { 24. La esposa está en el derecho de prohibirle a su esposo que trabaje o que estudie, si considera } \\
\text { que así él puede dedicarse mejor al hogar y/o a los hijos }\end{array}$ & $43(5.6 \%)$ & $4(3.1 \%)$ & 1.432 & .290 \\
\hline $\begin{array}{l}\text { 25. En ciertas situaciones se justifica que la esposa le prohíba a su esposo el trato con alguna } \\
\text { persona, como un amigo o una amiga }\end{array}$ & $234(30.5 \%)$ & $29(22.3 \%)$ & 3.607 & .061 \\
\hline
\end{tabular}


Continuación Tabla 3.

\begin{tabular}{|c|c|c|c|c|}
\hline $\begin{array}{l}\text { 26. Es comprensible que una esposa eche de la casa a su esposo si éste no ha cumplido con sus } \\
\text { deberes como padre }\end{array}$ & $198(25.8 \%)$ & $23(17.7 \%)$ & 3.950 & $.048^{*}$ \\
\hline $\begin{array}{l}\text { 27. Es comprensible que una esposa eche de la casa a su esposo si éste no ha cumplido con sus } \\
\text { deberes como cónyuge }\end{array}$ & $93(12.1 \%)$ & $14(10.8 \%)$ & .195 & .77 \\
\hline 28. Es una obligación del esposo satisfacer sexualmente a su esposa & $203(26.5 \%)$ & $24(18.5 \%)$ & 3.827 & $.05^{*}$ \\
\hline
\end{tabular}

Nota. $\mathrm{X}^{2}$ : Chi Cuadrada de Pearson. $* \mathrm{p} \leq .05$, dos colas $\quad * * \mathrm{p} \leq .01$, dos colas $\quad * * * \mathrm{p} \leq .001$, dos colas

para las necesidades de los hijos, a pesar de que era quien contaba con dinero para cubrir esas necesidades (de la madre al padre $)\left(\mathrm{X}^{2}[1,417]=6.763, \mathrm{p}=.005\right)$. Las mujeres del grupo VN, por su parte, solamente reportaron con mayor frecuencia haber presenciado la conducta de "Negarse a darle plata para las necesidades de los hijos, a pesar de que era quien contaba con dinero para cubrir esas necesidades" (de la madre al padre) $\left(\mathrm{X}^{2}[1,479]=4.795, \mathrm{p}=.028\right)$.

\section{DISCUSIÓN}

Esta investigación tuvo como objetivo comparar a un grupo de adolescentes y adultos jóvenes que ejercieron algún tipo de maltrato hacia su pareja en el noviazgo, con un grupo similar que no ejerció este tipo de violencia, en relación con el número de actos de violencia observados entre los padres; el número de acuerdos con afirmaciones a favor del uso de la violencia en el matrimonio; su puntuación en rasgos agresivos de personalidad, dominación e intransigencia y su puntuación en comunicación de pareja. Asimismo, se examinó la correlación entre estas variables y la frecuencia de realización de actos de malos tratos hacia la pareja.

En general, los resultados muestran, de manera similar a estudios previos, que la ejecución de malos tratos en el noviazgo por parte de adolescentes y adultos jóvenes parece estar relacionada con la observación de actos de maltrato entre los padres (e. g., Ackard \& Neumark-Sztainer, 2002; Carr \& Vandeusen, 2002; Foshee et al., 1999; Gagné et al., 2005; Kinsfogel \& Grych 2004; Rich et al., 2005; Rivera et al., 2007; Sears et al., 2007; Wolfe et al., 2001), la aceptación del uso de la violencia en la pareja (e. g., Carlson, 1990; Foshee et al., 1999) y rasgos de personalidad agresivos (e. g. Kinsfogel \& Grych, 2004).

Así, los participantes que ejercieron algún acto de maltrato hacia su pareja, observaron un número significativamente mayor de conductas de maltrato entre sus padres, tanto a nivel general como por sexo, aunque los tamaños del efecto obtenidos en estas comparaciones fueron bajos, lo que indica que esta variable no diferencia forzosamente a los individuos que han ejercido alguna conducta de maltrato a la pareja en el noviazgo de los que no. Esto lo confirma el hecho de que la fuerza de asociación entre estas dos variables fue baja, tanto a nivel general como por sexo, aunque estas correlaciones fueron estadísticamente significativas.

Los dos grupos también difirieron estadísticamente en sus puntuaciones en rasgos agresivos y en el número de enunciados a favor de la violencia en el matrimonio, aunque los tamaños del efecto obtenidos fueron bajos, lo que indica que estas variables no discriminan suficientemente a los individuos que han ejercido algún tipo de maltrato a su pareja en el noviazgo de los que no lo han hecho, particularmente entre los varones, ya que no se encontraron diferencias estadísticamente significativas entre el grupo de hombres VN y el grupo NVN. En ese sentido, es importante destacar que si bien estas dos variables correlacionaron positivamente con la frecuencia de malos tratos a nivel general y por sexo, dichas correlaciones también fueron bajas.

Los resultados fueron negativos en relación con la comunicación afectiva de pareja, ya que no se encontraron diferencias estadísticamente significativas entre los grupos en esta variable, y aunque ésta correlacionó negativamente con la frecuencia de malos tratos hacia la pareja, a nivel general y por sexo, dicha correlación fue baja. Por lo tanto, estos resultados sugieren que es necesario examinar nuevamente la relación entre estas dos variables, a pesar de que la comunicación de pareja ha sido considerada en algunos programas de prevención de los malos tratos en el noviazgo (Avery, Cascardi, O'leary \& Cano, 1997; Pick, Leenen, Givaudan \& Prado, 2010; Schwartz, Griffin, Russell \& Frontaura, 2006).

Los resultados referentes a los enunciados a favor de la violencia en el matrimonio, por otra parte, mostraron que los participantes que ejercieron al menos una conducta de maltrato hacia su pareja, estuvieron más frecuentemente de acuerdo con la realización de actos como los gritos, los insultos y las prohibiciones, por parte del esposo hacia la esposa para resolver conflictos relacionados con su papel como madre, así como con la ejecución de conductas similares por parte de la esposa hacia el marido para afrontar 
Tabla 4.

Diferencias entre el porcentaje de participantes que informaron al menos una conducta de maltrato de pareja y los que no, con respecto a las formas de violencia presenciadas entre los padres

\begin{tabular}{|c|c|c|c|c|c|}
\hline Violencia informada & Tipo & Maltrató & No maltrató & $\mathrm{X}^{2}$ & $\mathrm{p}$ \\
\hline 1. Actos de violencia física & $\mathrm{P}$ & $224(29.1 \%)$ & $22(16.9 \%)$ & 8.291 & $.004 * *$ \\
\hline 2. Actos de violencia física & M & $95(12.4 \%)$ & $11(8.5 \%)$ & 1.62 & .24 \\
\hline 3. Groserías, insultos o humillaciones (por lo menos una vez por semana) & $\mathrm{P}$ & $261(33.9 \%)$ & $25(19.2 \%)$ & 11.092 & $.001 * * *$ \\
\hline 4. Groserías, insultos o humillaciones (por lo menos una vez por semana) & M & $183(23.8 \%)$ & $16(12.3 \%)$ & 8.479 & $.003 * *$ \\
\hline 5. Amenaza verbal & $\mathrm{P}$ & $76(9.9 \%)$ & $8(6.2 \%)$ & 1.836 & .196 \\
\hline 6. Amenaza verbal & M & $34(4.4 \%)$ & $5(3.8 \%)$ & 0.09 & 1.0 \\
\hline 7. Amenaza con un arma u objeto que pueda causar daño & $\mathrm{P}$ & $57(7.4 \%)$ & $3(2.3 \%)$ & 4.664 & $.034^{*}$ \\
\hline 8. Amenaza con un arma u objeto que pueda causar daño & M & $23(3 \%)$ & $1(0.8 \%)$ & 2.117 & .235 \\
\hline 9. Encerrar en la casa o en una cuarto & $\mathrm{P}$ & $44(5.7 \%)$ & $3(2.3 \%)$ & 2.616 & .135 \\
\hline 10. Encerrar en la casa o en una cuarto & M & $81(10.5 \%)$ & $5(3.8 \%)$ & 5.748 & $.015^{*}$ \\
\hline 11. Prohibirle que trabaje o que estudie & $\mathrm{P}$ & $84(10.9 \%)$ & $11(8.5 \%)$ & 0.72 & .445 \\
\hline 12. Prohibirle que trabaje o que estudie & M & $73(9.5 \%)$ & $6(4.6 \%)$ & 3.326 & .092 \\
\hline 13. Prohibirle que salga a verse con un familiar o con un amigo o amiga & $\mathrm{P}$ & $28(3.7 \%)$ & $2(1.5 \%)$ & 1.554 & .295 \\
\hline 14. Prohibirle que salga a verse con un familiar o con un amigo o amiga & M & $50(6.5 \%)$ & $4(3.1 \%)$ & 2.328 & .162 \\
\hline 15. Echarla de la casa o cerrarle la puerta para que no pudiese entrar & $\mathrm{P}$ & $144(18.7 \%)$ & $11(8.5 \%)$ & 8.18 & $.004^{* *}$ \\
\hline 16. Echarlo de la casa o cerrarle la puerta para que no pudiese entrar & M & $28(3.6 \%)$ & $2(1.6 \%)$ & 1.5 & .295 \\
\hline 17. Obligarla a tener relaciones sexuales & $\mathrm{P}$ & $24(3.1 \%)$ & $1(0.8 \%)$ & 2.285 & .158 \\
\hline 18. Obligarlo a tener relaciones sexuales & M & $51(6.6 \%)$ & $2(1.5 \%)$ & 5.2 & $.024 *$ \\
\hline 19. Quitarle dinero u obligarla a que le diera dinero & $\mathrm{P}$ & $24(3.1 \%)$ & $0(0 \%)$ & 4.169 & $.037^{*}$ \\
\hline 20. Quitarle dinero u obligarlo a que le diera dinero & M & $7(0.9 \%)$ & $0(0 \%)$ & 1.183 & .602 \\
\hline $\begin{array}{l}\text { 21. Negarse a darle plata para las necesidades de los hijos, a pesar de que era } \\
\text { quien contaba con dinero para cubrir esas necesidades }\end{array}$ & $\mathrm{P}$ & $37(4.8 \%)$ & $1(0.8 \%)$ & 4.497 & $.032 *$ \\
\hline $\begin{array}{l}\text { 22. Negarse a darle plata para las necesidades de los hijos, a pesar de que era } \\
\text { quien contaba con dinero para cubrir esas necesidades }\end{array}$ & M & $93(12.1 \%)$ & $3(2.3 \%)$ & 10.983 & $.000^{* * *}$ \\
\hline 23. Amenazar con hacerle algo malo a los hijos & $\mathrm{P}$ & $4(0.5 \%)$ & $0(0 \%)$ & 0.683 & 1.0 \\
\hline 24. Amenazar con hacerle algo malo a los hijos & M & $34(4.4 \%)$ & $0(0 \%)$ & 5.99 & $.01 * *$ \\
\hline 25. Amenazar con hacerle algo malo a un familiar & $\mathrm{P}$ & $14(1.8 \%)$ & $1(0.8 \%)$ & 0.752 & .71 \\
\hline 26. Amenazar con hacerle algo malo a un familiar & M & $3(0.4 \%)$ & $1(0.8 \%)$ & 0.362 & .465 \\
\hline 27. Amenazar con llevarse a los hijos & $\mathrm{P}$ & $11(1.4 \%)$ & $0(0 \%)$ & 1.885 & .382 \\
\hline 28. Amenazar con llevarse a los hijos & M & $44(5.7 \%)$ & $3(2.3 \%)$ & 2.585 & .135 \\
\hline 29. Otras formas de violencia distintas & $\mathrm{P}$ & $63(8.2 \%)$ & $6(4.6 \%)$ & 1.998 & .211 \\
\hline 30. Otras formas de violencia distintas & M & $35(4.6 \%)$ & $2(1.5 \%)$ & 2.558 & .15 \\
\hline Total & & $458(59.4 \%)$ & $40(30.8 \%)$ & 36.894 & $.000 * * *$ \\
\hline
\end{tabular}

Nota. P: Del padre hacia la madre; M: De la madre hacia el padre.

$* \mathrm{p} \leq .05$, dos colas $* * \mathrm{p} \leq .01$, dos colas $\quad * * * \mathrm{p} \leq .001$, dos colas 
situaciones referentes a su papel como esposo y padre. Estos participantes también estuvieron más frecuentemente de acuerdo con la afirmación de que "la infidelidad del esposo es un motivo justificable para que su esposa le pegue", lo que sugiere, junto con los resultados anteriores, que la aceptación del uso de la violencia en el matrimonio podría relacionarse con los malos tratos en el noviazgo y debería ser otro objetivo de los programas de prevención de este tipo de violencia. No obstante, la aceptación de este tipo de conductas en el matrimonio parece ser mayor entre las mujeres que entre los hombres que han ejercido algún tipo de maltrato hacia su pareja en el noviazgo, ya que ellas estuvieron más de acuerdo, en un porcentaje significativamente mayor, con cuatro de los 28 enunciados analizados, uno referente al uso de la coerción y la violencia hacia el esposo, y los otros tres de la esposa al cónyuge, mientras que los varones que ejercieron algún tipo de maltrato hacia su pareja no presentaron diferencias estadísticamente significativas con respecto a los hombres que no maltrataron a su pareja.

Un análisis más preciso de los resultados evidencia algunas correspondencias entre las conductas de maltrato observadas entre los padres y los enunciados a favor del uso de la violencia en el matrimonio, dentro del grupo VN, ya que estos participantes presenciaron más frecuentemente agresiones verbales como groserías, insultos o humillaciones tanto del padre hacia la madre, como de la madre hacia el padre, y estuvieron más de acuerdo con el uso de los gritos por parte del esposo hacia la esposa y viceversa. Es decir que estos participantes presenciaron más frecuentemente diferentes formas de violencia verbal entre sus padres y, a su vez, estuvieron más de acuerdo con su utilización en el matrimonio. Estos participantes también informaron haber presenciado con más frecuencia cómo su madre obligaba a su padre a tener relaciones sexuales y, a su vez, estuvieron más de acuerdo con la afirmación de que es una obligación del padre satisfacer sexualmente a su esposa.

De esta manera, los resultados generales obtenidos en esta investigación tienden a ser coherentes con la noción de que el aprendizaje social tiene un papel importante en el ciclo inter-generacional de la violencia desde la adolescencia y sugieren tentativamente que en la evaluación del riesgo de maltrato hacia la pareja en el noviazgo, así como en la prevención secundaria y el tratamiento de este tipo de violencia, debería contemplarse la observación de violencia en la familia de origen, así como los rasgos de personalidad y las actitudes a favor de la violencia. Particularmente, estos resultados tienden a sugerir la conveniencia de contemplar en el tratamiento y la prevención de la violencia en el noviazgo, un proceso educativo sobre los tipos y formas de maltrato hacia la pareja, la influencia del entorno socio-familiar, una adecuada preparación para establecer relaciones de pareja antes y en el matrimonio y la importancia de confrontar las creencias que justifican el uso de la violencia en la pareja, tal como se ha efectuado en programas como los desarrollados por Avery et al. (1997), Bruce, Nan \& Frances (2010), Gómez (2007), Pick, Leenen, Givaudan \& Prado (2010) y Schwartz, Griffin, Russell \& Frontaura (2006).

Este estudio presenta como principales fortalezas el uso de una muestra grande de participantes, así como el análisis de un conjunto relativamente amplio de variables y la realización de estos análisis, tanto a nivel general como por sexo. Sin embargo, tiene algunas limitaciones que deberán ser tenidas en cuenta en futuros estudios, ya que los participantes no se distribuían uniformemente con respecto a la edad y al estrato socioeconómico, lo que dificulta la generalización de estos resultados a adolescentes de menor edad y adultos de mayor edad, o que tienen otros niveles educativos y socioeconómicos. Por otra parte, el instrumento con el que se recogió información sobre los malos tratos en el noviazgo no contemplaba dimensiones como la severidad o la duración de estos malos tratos, por lo que sería conveniente que en otras investigaciones se tuvieran en cuenta estos aspectos.

\section{REFERENCIAS}

Ackard, D. M., \& Neumark-Sztainer, D. (2002). Date Violence and Date Rape among Adolescents: Associations with Disordered Eating Behaviors and Psychological Health. Child Abuse \& Neglect, 26, 455-473.

Arroyo, C., y Vergara, H. (2000). Relación entre la valoración de la historia de maltrato físico y la comunicación afectiva en el noviazgo en adolescentes. Tesis de grado no publicada, Universidad de los Andes, Bogotá, Colombia.

Avery, L., Cascardi, M., O’leary, M., \& Cano (1997). Efficacy of a Dating Violence Prevention Program on Attitudes Justifying Aggression. Journal of Adolescence, 21(1), 11-17.

Bem, S. L. (1974). The Measurement of Psychological Androgeny. Journal of Counseling and Clinical Psychology, 42, 155-162.

Browne, K., \& Herbert M. (1997). Preventing Family Violence. Chichester: Wiley.

Bruce, T., Nan, S., \& Frances, B. (2010). The Effects of Gender Violence/Harassment Prevention Programming in Middle Schools: A Randomized Experimental Evaluation. Violence and Victims, 21(2), 202-223.

Carlson, B. E. (1990). Adolescent Observers of Marital Violence. Journal of Family Violence, 5(4), 285-299.

Carr, J. L., \& Vandeusen, K. M. (2002). The Relationship between Family of Origin Violence and Dating Violence 
in College Men. Journal of Interpersonal Violence, 17(6), 630-646.

Centers for Disease Control and Prevention (2006). Physical Dating Violence among High School Students, United States, 2003. Morbidity and Mortality Weekly Report, 55(19), 532-535.

Corral, S. (2009). Estudio de la violencia en el noviazgo en jóvenes universitarios/as: Cronicidad, severidad y mutualidad de las conductas violentas. Psicopatología Clínica Legal y Forense, 9, 29-48.

Foshee, V. A., Bauman, K. E., \& Linder, G. F. (1999). Family Violence and the Perpetration of Adolescent Dating Violence: Examining Social Learning and Social Control Processes. Journal of Marriage and the Family, 61(2), 331342.

Fredland, N. M., Ricardo, I. B., Canpbell, J. C., Sharps, P. W., et al. (2005). The Meaning of Dating Violence in the Lives of Middle School Adolescents: A Report Of A Focus Group Study. Journal of School Violence, 4(2), 95-114.

Gagné, M., Lavoie, F., \& Hébert, M. (2005). Victimization during Childhood and Revictimization in Dating Relationships in Adolescent Girls. Child Abuse \& Neglect, 29(10), 1155-1172.

Gómez, H. (2007). La prevención de la violencia de género en adolescentes. Una experiencia en el ámbito educativo. Apuntes de Psicología, 25(3), 325-340.

Howard, D. E., \& Wang, M. Q. (2003). Risk Profiles of Adolescent Girls Who Were Victims of Dating Violence. Adolescence, 38(149), 1-14.

Kinsfogel, K. M., \& Grych, J. H. (2004). Interparental Conflict and Adolescent Dating Relationships: Integrating Cognitive, Emotional, and Peer Influences. Journal of Family Psychology, 18(3), 505-515.

Kreiter, S., Krowchuk, D. P., Woods, C. R., Sinal, S. H., Lawless, M. R., \& Durant, R. H. (1999). Gender Differences in Risk Behaviors among Adolescents Who Experience Date Figthing. Pediatrics, 104(6), 1286-1292.

Lara, M. A. (1993). Inventario de Masculinidad y Feminidad. México: Manual Moderno.

Lewis, S. F., \& Fremouw, W. (2001). Dating Violence: A Critical Review of the Literature. Clinical Psychology Review, 21(1), 105-127.

Lichter, E. L., \& McCloskey, L. A. (2004). The Effects of Childhood Exposure to Marital Violence on Adolescent Gender-Role Beliefs and Dating Violence. Psychology of Women Quarterly, 28(4), 344-357.

Macbeth, G., Razumiejczyk, E., \& Ledesma, R. D. (2001). Cliff's Delta Calculator: A Non-Parametric Effect Size Program for Two Groups of Observations. Universitas Psychologica, 10(2), 545-555.

Martínez-Gómez, J. A., Guerrero-Rodríguez, S. I., y ReyAnacona, C. A. (2012). Evaluación de la validez de constructo y la confiabilidad del Inventario de Masculinidad y Femineidad en adolescentes y adultos jóvenes colombianos. Avances en Psicología Latinoamericana, 30(1), 170-181.

Matud, M. P. (2007). Dating Violence and Domestic Violence (Editorial). Journal of Adolescent Health, 40, 295-297.

Mihalic, S. W., \& Elliot, D. (1997). A Social Learning Theory Model of Marital Violence. Journal of Family Violence, 12(1), 21-47.

Muñoz-Rivas, M. J., Gámez-Guadix, M., Graña, J. L., y Fernández, L. (2010). Violencia en el noviazgo y consumo de alcohol y drogas ilegales entre adolescentes y jóvenes españoles. Adicciones, 22(2), 125-134.

Pick, S., Leenen, L., Givaudan, M., y Prado, A. (2010). "Yo quiero, yo puedo... prevenir la violencia": Programa breve de sensibilización sobre violencia en el noviazgo. Salud Mental, 33, 153-160.

Rey-Anacona, C. A. (2008a). Prevalencia, factores de riesgo y problemáticas asociadas con la violencia en el noviazgo: Una revisión de la literatura. Avances en Psicología Latinoamericana, 26(2), 227-241.

Rey-Anacona, C. A. (2008b). Habilidades pro sociales, rasgos de personalidad de género y aceptación de la violencia hacia la mujer, en adolescentes que han presenciado violencia entre sus padres. Acta Colombiana de Psicología, 11(1), 107-118.

Rey Anacona, C. A. (2009a). Maltrato en el noviazgo de tipo físico, psicológico, emocional, sexual y económico: Un estudio exploratorio. Acta Colombiana de Psicología, 12(2), 27-36.

Rey-Anacona, C. A. (2009b). Características psicológicas de adolescentes varones $y$ adolescentes mujeres victimarios de violencia en su relación de noviazgo [informe de investigación]. Universidad Pedagógica y Tecnológica de Colombia.

Rey-Anacona, C. A., Mateus-Cubides, A. M., y BayonaArévalo, P. A. (2010). Malos tratos ejercidos por adolescentes durante el noviazgo: Diferencias por sexo. Revista Mexicana de Psicología, 27(2), 169-181.

Rich, C. L., Gidycz, Ch. A., Warkentin, J. B., Lohe, C., \& Weiland, P. (2005). Child and Adolescent Abuse and Subsequent Victimization: A Prospective Study. Child Abuse \& Neglect, 29(12), 1373-1394.

Rivera, L., Allen, B., Rodríguez, G., Chávez, R. y Lazcano, E. (2006). Violencia durante el noviazgo, depresión y conductas de riesgo en estudiantes femeninas (12-24 años). Salud Pública de México, 48(2), 288-296.

Rivera, L., Allen, B., Rodríguez, G., Chávez, R., y Lazcano, E. (2007). Prevalence and Correlates of Adolescent Dating Violence: Baseline Study of a Cohort of 7960 Male and Female Mexican Public School Students. Preventive Medicine, 44(6), 477-484.

Roberts T. A., Auinger, P., \& Klein J. D. (2006). Predictors of Partner Abuse in a Nationally Representative Sample of 
Adolescents Involved in Heterosexual Dating Relationships. Violence and Victims, 21(1), 81-89.

Saldivia, C., y Vizcarra, B. (2012). Consumo de drogas y violencia en el noviazgo en estudiantes universitarios del sur de Chile. Terapia Psicológica, 30(2), 43-49.

Schwartz, J., Griffin, L., Russell, M., \& Frontaura, S. (2006). Prevention of Dating Violence on College Campuses: An Innovative Program. Journal of College Counseling, 9, 9096.

Sears, H. A., Byers, E. S., \& Price, E. L. (2007). The Cooccurrence of Adolescent Boys' and Girls' Use of Psychologically, Physically, and Sexually Abusive Behaviours in Their Dating Relationships. Journal of Adolescence, 30(3), 487-504.

Silverman, J. G., Raj, A., Mucci, L. A., \& Hathaway, J. E. (2001). Dating Violence Against Adolescent Girls and
Associated Substance Use, Unhealthy Weight Control, Sexual Risk Behavior, Pregnancy, and Suicidality. Journal of the American Medical Association, 286, 572-579.

Smith, A., Winokur, K., \& Palenski, J. (2005). What is Dating Violence? An Exploratory Study of Hispanic Adolescent's Definitions. Journal of Ethnicity in Criminal Justice, $3(1 / 2), 1-20$.

Vizcarra, M. B., y Póo, A. M. (2011). Violencia de pareja en estudiantes universitarios del sur de Chile. Universitas Psychologica, 10(1), 89-98.

Wolfe, D. A., Scott, K., Wekerle, C., \& Pittman, A. (2001). Child Maltreatment: Risk of Adjustment Problems and Dating Violence In Adolescence. Journal of the American Academy of Child \& Adolescent Psychiatry, 40(3), 282-289. 